Revista Posgrado y Sociedad

Sistema de Estudios de Posgrado

Universidad Estatal a Distancia

ISSN 2215-2172

Costa Rica

revistaposgradoysoci@uned.ac.cr

\title{
Creencias sobre la clase, y el docente de matemáticas en la educación secundaria
}

Beliefs about the class and the teacher of Mathematics in secondary education

Eugenio Rojas M.

Ronald Sequeira S.

Escuela de Ciencias Exactas y Naturales, UNED

Volumen 12, Número 1

Marzo 2012

pp. 68-81

Recibido: Noviembre, 2011

Aprobado: Febrero, 2012 


\begin{abstract}
Resumen
Existen muchas opiniones por parte de estudiantes de secundaria sobre la clase y el docente de matemáticas, algunas positivas y otras no. Por ejemplo, para algunos las lecciones de matemáticas son aburridas, y muy difíciles. Para otros, las clases son interesantes y entretenidas. Con respecto al docente de Matemática, es aburrido, poco innovador, hábil con los números y responsable. En este artículo se describen algunas de las creencias que tienen los estudiantes sobre las clases y el docente de Matemática, como parte del Proyecto de Investigación: "Estructura de las Creencias en Matemática", con estudiantes de secundaria de tres instituciones de Costa Rica, dos de carácter público y una de carácter privado.
\end{abstract}

Palabras clave: creencias, educación Matemática, lección de matemáticas, docente de matemática, educación secundaria.

\begin{abstract}
There are many opinions from High School students about the class and the teacher of Mathematics, positive and negative. For example, some Mathematics lessons are boring and very difficult. For others, the classes are interesting and entertaining. With respect to teacher Math, they indicate that is boring, not very innovative, very strict, good with numbers and responsible. This article describes some of the beliefs that students have about the classes and the teaching of Mathematics, as part of Research Project "Structure of Beliefs in Mathematics" with High School students from three institutions in Costa Rica, two public and one private.
\end{abstract}

Keywords: beliefs, education, math, math lesson, math teacher, secondary education. 


\section{Introducción}

Muchos estudiantes tienen la creencia de que la clase de Matemática es la que tiene que ver con el trabajo con números, variables, funciones, y con lo que conocemos de geometría.

Efectivamente, en esta clase se trabaja con variables, funciones y geometría, sin embargo, es más que eso, pues además de tener entre sus objetivos que el alumno adquiera una serie de competencias en matemática, debe ser un espacio social en el que los estudiantes tengan libertad con responsabilidad y donde el docente propicie actividades de aprendizaje que puedan llevarse a cabo en un ambiente de colaboración y respeto.

También debe ser el lugar, donde los alumnos tengan la oportunidad de expresar sin temor a represalias, sus ideas $\mathrm{y}$ pensamientos, analicen $\mathrm{y}$ conjeturen sobre los problemas de su entorno inmediato y puedan entender cómo la matemática es una herramienta útil que les permite dar posibles soluciones a estos problemas.

De esta manera, la clase de Matemática tiene que ser un espacio de comunicación de ideas que, ya sea en forma oral o escrita, facilite el aprendizaje de esta disciplina y promueva la comprensión y adquisición de los contenidos aprehendidos, de manera tal que permita al estudiante contextualizar el conocimiento y transformar su propia realidad.

Para lograr lo anterior, la organización del trabajo en el aula y el rol del docente deben cambiar, pues se tiene que propiciar el trabajo en equipo para resolver los problemas del entorno, discutir en forma grupal sus conjeturas, estrategias de resolución y posibles soluciones de la situación problemática en estudio.

Así entonces, la mediación pedagógica del docente deberá estar orientada a facilitar la intercomunicación entre todos los actores del proceso educativo, con la finalidad de que por medio del razonamiento, los alumnos puedan tener una mejor comprensión de su propia realidad como seres que interactúan con otros, dentro de un complejo llamado sociedad.

De esta manera, algunas de las tareas que deberá asumir el nuevo docente serán:

$\checkmark$ Seleccionar problemas y actividades que tengan que ver con el entorno de los alumnos.

$\checkmark$ Promover problemas y contraejemplos que contradigan las hipótesis de los estudiantes, favoreciendo la reflexión y el dialogo.

$\checkmark$ Ser fuente de información para relacionar los conceptos y procedimientos propuestos por los estudiantes con el lenguaje convencional y formal.

$\checkmark$ Orientar el trabajo de los estudiantes, motivándolos a que participen en las diferentes actividades e informando el progreso alcanzado por cada uno de ellos.

En esta investigación se analizarán solamente las creencias que tienen los estudiantes sobre la clase de Matemática, el profesor de dicha asignatura y el rendimiento en esta materia.

El documento está estructurado de la siguiente forma: se hace una breve reseña de las creencias que existen en relación con la matemática, se describe la metodología empleada, se analizan los resultados obtenidos y finalmente se dan las conclusiones. 


\section{Creencias en Matemática}

La mayoría de las personas, asumen la visión de que las matemáticas tienen que ver con la exactitud y que saber matemáticas es tener habilidad en resolver ejercicios de aritmética, geometría, lógica y resolución de problemas. Esta mirada es eminentemente cultural y se refiere principalmente a la forma como nos la enseñan en la escuela, donde por lo general se sigue una serie de reglas dadas por el profesor y que deben ser aplicadas en forma mecánica en la resolución de los ejercicios. Allan Schoenfeld, citado por Barrantes (2008) dice que

Las creencias de cómo hacer matemáticas y qué significan en la escuela se adquieren a través de años, observando, escuchando $y$ practicando. (2008. p. 51)

Barrantes menciona que las creencias de estudiantes en relación con la matemática tienen que ver con el contexto del aula, el papel del docente, sus compañeros de clase y consigo mismo.

Por otra parte, para Pólya, (1966) las matemáticas son una construcción social donde los estudiantes discuten, conjeturan, analizan, y cuyos resultados deben evaluarse en relación con el entorno del estudiante.

Allan Schoenfeld retomando las investigaciones de Pólya sobre resolución de problemas, considera que el sistema de creencias es una forma muy particular que tienen los estudiantes de ver el mundo, de aproximarse a él y de determinar la manera de cómo enfrentar los problemas, así como el diseño de las estrategias de solución y las posibles respuestas que encontrará. De igual manera, otras investigaciones se han centrado en cuestiones de género y su influencia en el rendimiento académico como lo señala Gómez (2007).

A partir de la década de los 90, las creencias en matemática adquieren importancia para los científicos en Educación Matemática como Schoenfeld, De Corte, Verschaffel, entre otros, según lo cita Gómez (2007). Como resultado de todas estas investigaciones, los científicos en Educación Matemática, han determinado cinco categorías aptitudinales que debe poseer el estudiante para tener una buena disposición hacia la matemática; una de ellas se refiere a las creencias positivas sobre esta disciplina y su aprendizaje.

En la actualidad, las ciencias sociales reconocen la importancia de las creencias que tienen los estudiantes sobre la matemática, como un elemento que hay que considerar en el proceso de enseñanza y aprendizaje de esta disciplina. No obstante, lo que ha faltado es evaluar estas creencias en el propio contexto del estudiante como lo sería su familia, comunidad, institución educativa, e inclusive, las creencias que tenga el docente con respecto a esta disciplina.

Más aún, De Corte y Eynde (2002) señalan que las creencias de los estudiantes sobre las matemáticas, están determinadas por el contexto social donde interactúan y por sus necesidades psicológicas individuales.

Estos autores afirman que los sistemas de creencias de los estudiantes están constituidos por las creencias sobre la 
educación matemática, creencias sobre el contexto y creencias sobre sí mismos. Entre las creencias sobre la educación matemática se considera las creencias de los estudiantes sobre la matemática, la enseñanza y el aprendizaje y la resolución de problemas; y entre las creencias de los estudiantes sobre el contexto se considera las creencias sobre el papel del docente y el de los compañeros de clase.

De igual manera, De Faria (2008) indica que la experiencia que ha tenido un estudiante al aprender matemáticas, provoca en él distintas reacciones emocionales que determinan de una $u$ otra forma su sistema de creencias y que influyen en su capacidad para aprender, determinando una relación cíclica entre creencias y aprendizaje.

Pehkonen y Törner, 1996, citados por el mismo De Faria (2008), afirman que las creencias impactan en forma significativa la forma en que los estudiantes aprenden y utilizan las matemáticas; si sus creencias son negativas hacia la matemáticas, entonces su aprendizaje será pasivo, enfatizando el uso de la memoria para su comprensión.

Por otra parte, Grossman, Wilson y Shulman, citados por (Llinares, 1990) hacen la diferencia entre creencias y conocimiento, en el sentido de que las primeras están más abiertas al debate y no siguen cánones de evidencia utilizados en el conocimiento.

Flores, citado por De Faria (2008. p.18) dice que "las creencias matemáticas son significados que se atribuyen a las matemáticas, a su enseñanza y al aprendizaje de las mismas".

Del mismo modo, Moreno y Azcárate, citados por el mismo autor, manifiestan que las creencias son:

Conocimientos subjetivos poco elaborados, generados a nivel particular por cada individuo para explicarse y justificar muchas de las decisiones $y$ actuaciones personales $y$ profesionales vividas. Moreno $\mathrm{y}$ Azcárate, (2003. p. 10).

Es importante acotar que el concepto creencias en el campo de la educación se ha utilizado en forma indiscriminada, inclusive se usan sinónimos para referirse a éste término como: concepciones, ideas, valores y actitudes, más aún, en el ámbito propiamente de la educación matemática, las creencias y el conocimiento se ha contextualizado principalmente en el currículo y en el proceso de enseñanza y aprendizaje.

Ahora bien, es un hecho que el paradigma mecanicista de Descartes, adoptado por el mundo desde el siglo XVII no responde a las inquietudes y necesidades del ser humano en este nuevo siglo, por eso es que debe surgir un nuevo orden social que dé respuestas y satisfaga esas necesidades, este nuevo orden, es precisamente el nuevo paradigma o paradigma emergente.

Dos de los principios de este nuevo paradigma establecen que las creencias crean nuestra propia realidad a partir de lo que entendemos más profundamente como verdadero y que 
estos sistemas crean realidades independientes, autosuficientes, autoduraderas y auto-realizables.

De acuerdo con lo anterior, se asume en este artículo el sistema de creencias desde los principios del Nuevo Paradigma, dado por Red Armonía Mundial:

Un sistema de creencias es el que crea su propia realidad independiente, auto-suficiente, auto-durable y autorealizable, que es completa en sí misma $y$ ofrece todas las evidencias para soportarla y hacerla real de una manera autónoma y autosuficiente. (2003. p. 2)

\section{Metodología}

En esta investigación se utilizó una metodología de tipo cuantitativo que permite conocer la opinión de los estudiantes de secundaria sobre las creencias que tienen sobre la matemática.

El diseño general de la metodología fue la selección de las categorías para el cuestionario, seleccionar la población, elaboración, validación y aplicación del instrumento, análisis de los cuestionarios, discusión de los resultados y conclusiones.

En este artículo solamente se analiza la información concerniente a la opinión dada por los estudiantes con respecto a la clase y al docente de matemática, así como si el rendimiento en esta disciplina depende de la actitud que tiene con respecto al docente. Para ello, el estudiante respondió cuatro preguntas, dos de tipo cerrado y dos abiertas que se describen en el apartado sobre la elaboración de instrumentos. Descripción de la población
La población está constituida por estudiantes de educación secundaria de tres instituciones educativas de Costa Rica: el Liceo de Atenas, ubicado en la provincia de Alajuela, el Liceo San Isidro en Heredia y el Colegio Calasanz en la capital, San José.

Los estudiantes de la investigación se encontraban matriculados en octavo y décimo año, respectivamente. Hay que señalar que las tres instituciones tienen matrícula mixta y que de acuerdo con el Ministerio de Educación Pública su modalidad es académica diurna; siendo solamente el Colegio Calasanz de carácter privado, mientras que las otras dos, son públicas financiadas por el Estado costarricense.

Por otra parte, el Liceo de Atenas por las características geográficas donde se localiza, se considera una institución que se ubica en la zona rural del país, no así los otros dos colegios que están localizados en el Gran Valle Central. Los alumnos que asisten al Liceo de Atenas y al Colegio San Isidro pertenecen a las clases sociales baja y media del país, mientras que los del Colegio Calasanz se ubican en la clase social media- alta.

\section{Elaboración de los instrumentos}

El instrumento utilizado consta de dos partes, la primera con preguntas de tipo cerrado con 26 proposiciones relacionadas con la matemática donde los estudiantes tienen que dar su opinión a partir de cinco categorías que surgen de la escala de Likert que se describe a continuación:

$\mathbf{1}=$ totalmente de acuerdo

$2=$ de acuerdo

$3=$ ni de acuerdo ni en desacuerdo

$4=$ en desacuerdo 
$5=$ totalmente en desacuerdo

Las preguntas de tipo cerrado seleccionadas del cuestionario que tienen que ver con la opinión de los estudiantes sobre la clase de matemática y su rendimiento académico se observan en la tabla 1.

Tabla 1. Preguntas de tipo cerrado

\begin{tabular}{|l|l|ll|}
\hline 18 & $\begin{array}{l}\text { Las clases de Matemáticas son eternas y } \\
\text { pesadas. }\end{array}$ & $\begin{array}{l}\text { Mi rendimiento en Matemáticas depende } \\
\text { en gran medida de la actitud del profesor } \\
\text { hacia mí }\end{array}$ & \\
\hline 19
\end{tabular}

De igual forma, la segunda parte del instrumento consta de 6 preguntas abiertas que tienen que ver sobre la matemática, la clase de matemática, el profesor de matemática, el saber matemática, mis capacidades en matemáticas y el uso de la calculadora.

Las preguntas abiertas que fueron seleccionadas son las siguientes:

28. La clase de Matemática es:

29. Mi profesor de Matemática es:

Finalmente, cabe señalar que el instrumento fue validado con estudiantes de décimo año de otras instituciones y con estudiantes de octavo año del Liceo de Puriscal.

\section{Procedimiento}

Después de aplicar el cuestionario se procedió a la obtención de los datos y al tratamiento de la información de la siguiente manera:

1. Se tabuló la información de las dos preguntas cerradas en la hoja electrónica Excel.

2. Se enlistó las opiniones de los estudiantes con respecto a las dos preguntas abiertas y se hizo una codificación para simplificar el proceso de análisis y poder contrastar esta información con la obtenida en las dos preguntas abiertas.

4. Finalmente se realiza el estudio y análisis de la información, la cual se presenta mediante tablas.

\section{Análisis de resultados}

En total se encuestaron a 219 estudiantes cuya distribución por institución, sexo y nivel se muestran en la tabla 2. 


\begin{tabular}{|c|c|c|c|c|c|c|c|c|c|c|c|c|}
\hline \multicolumn{13}{|c|}{$\begin{array}{c}\text { Tabla } \mathbf{2} \\
\text { Distribución de la población según sexo y nivel que cursa }\end{array}$} \\
\hline \multirow[t]{3}{*}{ Institución } & \multirow{2}{*}{\multicolumn{2}{|c|}{$\begin{array}{l}\text { Octavo } \\
\text { Masc. }\end{array}$}} & \multirow{2}{*}{\multicolumn{2}{|c|}{$\begin{array}{l}\text { Décimo } \\
\text { Masc. }\end{array}$}} & \multirow{2}{*}{\multicolumn{2}{|c|}{$\begin{array}{l}\text { Octavo } \\
\text { Feme. }\end{array}$}} & \multirow{2}{*}{\multicolumn{2}{|c|}{$\begin{array}{l}\text { Décimo } \\
\text { Feme. }\end{array}$}} & \multicolumn{4}{|c|}{ Total } \\
\hline & & & & & & & & & & c. & & ne. \\
\hline & $A$ & $\%$ & $A$ & $\%$ & $A$ & $\%$ & $A$ & $\%$ & $A$ & $\%$ & $A$ & $\%$ \\
\hline $\begin{array}{l}\text { Liceo de } \\
\text { Atenas }\end{array}$ & 18 & 30 & 17 & 33 & 14 & 25 & 16 & 31 & 35 & 32 & 30 & 28 \\
\hline $\begin{array}{l}\text { Colegio } \\
\text { Calasanz }\end{array}$ & 18 & 30 & 16 & 32 & 19 & 34 & 17 & 33 & 34 & 30 & 36 & 33 \\
\hline $\begin{array}{l}\text { Liceo de San } \\
\text { Isidro }\end{array}$ & 24 & 40 & 18 & 35 & 23 & 41 & 19 & 36 & 42 & 38 & 42 & 39 \\
\hline Total & 60 & 100 & 51 & 100 & 56 & 100 & 52 & 100 & 111 & 100 & 108 & 100 \\
\hline
\end{tabular}

Fuente: Estadísticas Proyecto Creencias en Matemática

En cuanto al sexo de la población participante, el 50,7\% (111 estudiantes) son hombres $y$ el $49,3 \% \quad(108$ estudiantes) son mujeres, siendo el Liceo de San Isidro el que tiene la mayor población.

Por otro lado, el 53\% (116 estudiantes) está matriculado en octavo año y el $47 \%$ (103 estudiantes) en décimo, siendo también el Liceo de San Isidro con más estudiantes en octavo y décimo respectivamente.

Análisis de la pregunta: Las clases de matemáticas son eternas y pesadas.

En la tabla 3 se muestran las opiniones de los estudiantes según institución y nivel que cursan con respecto a si las clases de matemáticas son eternas y pesadas.

Tabla 3

\begin{tabular}{|c|c|c|c|c|c|c|c|c|c|c|c|c|c|c|}
\hline & nió & de I & es & udia & $\operatorname{tes}$ & $\begin{array}{l}\text { Tab } \\
\text { on re }\end{array}$ & 3 & a la & clas & de $\mathrm{M}$ & aten & lática & & \\
\hline Categorías & Lic & eo d & Ate & nas & Co & egio & Cala: & $\operatorname{sanz}$ & Lic & eo Sa & n Is & idro & & tal \\
\hline & $\mathbf{O C}$ & avo & Dé & imo & Oc & avo & Déc & imo & Oc & avo & Dée & imo & & \\
\hline & $A$ & $\%$ & $A$ & $\%$ & $A$ & $\%$ & $A$ & $\%$ & $A$ & $\%$ & $A$ & $\%$ & $A$ & $\%$ \\
\hline $\begin{array}{l}\text { Totalmente } \\
\text { de acuerdo }\end{array}$ & 2 & 6 & 2 & 6 & 6 & 16 & 5 & 15 & 4 & 8 & 2 & 5 & 21 & 10 \\
\hline De acuerdo & 7 & 22 & 4 & 12 & 10 & 27 & 6 & 18 & 6 & 13 & 5 & 14 & 38 & 17 \\
\hline $\begin{array}{l}\mathrm{Ni} \mathrm{de} \\
\text { acuerdo ni } \\
\text { en } \\
\text { desacuerdo }\end{array}$ & 12 & 38 & 5 & 15 & 12 & 32 & 10 & 31 & 26 & 55 & 14 & 38 & 79 & 36 \\
\hline $\begin{array}{l}\text { En } \\
\text { desacuerdo }\end{array}$ & 6 & 18 & 3 & 9 & 5 & 14 & 6 & 18 & 4 & 9 & 7 & 19 & 31 & 14 \\
\hline $\begin{array}{l}\text { Totalmente } \\
\text { en } \\
\text { desacuerdo }\end{array}$ & 5 & 16 & 19 & 58 & 4 & 11 & 6 & 18 & 7 & 15 & 9 & 24 & 50 & 23 \\
\hline Total & 32 & 100 & 33 & 100 & 37 & 100 & 33 & 100 & 47 & 100 & 37 & 100 & 219 & 100 \\
\hline
\end{tabular}

Fuente: Estadísticas Proyecto Creencias en Matemática. 
Según la tabla 3 , se observa que el $37 \%$ (81 estudiantes) está "totalmente en desacuerdo" y "en desacuerdo" en que las clases de Matemáticas son eternas y pesadas, mientras que el 36\% (79 estudiantes) está "ni de acuerdo ni en desacuerdo". De igual forma el $27 \%$ (59 estudiantes) manifiesta que está "de acuerdo" y "totalmente de acuerdo" con esta afirmación.

También se observa que en el Liceo de Atenas, el 67\% (22 estudiantes de la población de décimo año de ese colegio) opinan que las clases de
Matemáticas no son eternas ni pesadas, caso contrario en el Liceo de San Isidro, donde el 55\% (26 estudiantes de la población de octavo año de ese colegio) y el 38\% (14 estudiantes de la población de décimo año de ese colegio), están ni de acuerdo ni en desacuerdo con esta opinión.

En la tabla 4 se resumen las opiniones de los estudiantes con respecto a esta pregunta según sexo y nivel que cursan.

\begin{tabular}{|l|c|c|c|c|c|c|c|c|c|c|c|c|c|}
\hline \multicolumn{10}{|c|}{ Tabla 4 } \\
\multicolumn{10}{|c|}{ Opinión de los estudiantes sobre las clases de Matemáticas, según sexo y nivel que } \\
cursan. \\
\hline
\end{tabular}

Si se analiza la opinión de los estudiantes según el nivel matriculado, se tiene que el 48,5\% (50 estudiantes) de los alumnos de décimo año (103 estudiantes), está "totalmente en desacuerdo" y "en desacuerdo" en que las clases de Matemáticas son eternas y pesadas, mientras que el 30\% (35 estudiantes) de los alumnos de octavo año (116 estudiantes), está "de acuerdo" y "totalmente de acuerdo" con esta afirmación. De igual forma, el 36\% (79 estudiantes) del total de la población manifiesta que está "ni de acuerdo" ni en desacuerdo" con esta afirmación.

Por otro lado, considerando la opinión de los estudiantes, según sexo, se puede notar que el 42\% (47 estudiantes) de los hombres (111 estudiantes) está "totalmente en desacuerdo" y "en desacuerdo" en que las lecciones de Matemáticas son eternas y pesadas, y de las 108 mujeres el $31 \%$ (34 estudiantes) coincide en esta categorización. 
Análisis de la pregunta: Mi rendimiento en Matemáticas depende en gran medida de la actitud del profesor hacia mí.

En la tabla 5 se muestran las opiniones de los estudiantes según institución y nivel que cursan con respecto a sí su rendimiento académico depende de la actitud del profesor hacia él o ella.

\begin{tabular}{|c|c|c|c|c|c|c|c|c|c|c|c|c|c|c|c|c|}
\hline \multirow{2}{*}{\multicolumn{5}{|c|}{\begin{tabular}{|l}
\multicolumn{2}{|c}{ Opinión de los estudiantes con } \\
Categorías \\
Liceo de Atenas
\end{tabular}}} & \multicolumn{12}{|c|}{$\begin{array}{l}\text { Tabla } \mathbf{5} \\
\text { rendimiento académico en relación con la actitud del } \\
\text { esor hacia ellos. }\end{array}$} \\
\hline & & & & & \multicolumn{4}{|c|}{ Colegio Calasanz } & \multicolumn{4}{|c|}{ Liceo San Isidro } & \multicolumn{4}{|c|}{ Total } \\
\hline & \multicolumn{2}{|c|}{ Octavo } & \multicolumn{2}{|c|}{ Décimo } & \multicolumn{2}{|c|}{ Octavo } & \multicolumn{2}{|c|}{ Décimo } & \multicolumn{2}{|c|}{ Octavo } & \multicolumn{2}{|c|}{ Décimo } & \multicolumn{2}{|c|}{ Octavo } & \multicolumn{2}{|c|}{ Décimo } \\
\hline & A & $\%$ & $A$ & $\%$ & $A$ & $\%$ & $A$ & $\%$ & $A$ & $\%$ & $A$ & $\%$ & $A$ & $\%$ & $A$ & $\%$ \\
\hline $\begin{array}{l}\text { Totalmente } \\
\text { de acuerdo }\end{array}$ & 6 & 19 & 5 & 15 & 11 & 30 & 9 & 28 & 11 & 23 & 8 & 22 & 28 & 24 & 22 & 21 \\
\hline De acuerdo & 3 & 9 & 7 & 21 & 9 & 24 & 5 & 15 & 10 & 21 & 8 & 22 & 22 & 19 & 20 & 20 \\
\hline $\begin{array}{l}\text { Ni de } \\
\text { acuerdo ni } \\
\text { en } \\
\text { desacuerdo }\end{array}$ & 10 & 31 & 9 & 28 & 10 & 27 & 7 & 21 & 17 & 36 & 7 & 19 & 37 & 32 & 23 & 22 \\
\hline $\begin{array}{l}\text { En } \\
\text { desacuerdo }\end{array}$ & 5 & 16 & 7 & 21 & 4 & 11 & 8 & 24 & 3 & 7 & 11 & 29 & 12 & 10 & 26 & 25 \\
\hline $\begin{array}{l}\text { Totalmente } \\
\text { en } \\
\text { desacuerdo }\end{array}$ & 8 & 25 & 5 & 15 & 3 & 8 & 4 & 12 & 6 & 13 & 3 & 8 & 17 & 15 & 12 & 12 \\
\hline Total & 32 & 100 & 33 & 100 & 37 & 100 & 33 & 100 & 47 & 100 & 37 & 100 & 116 & 100 & 103 & 100 \\
\hline
\end{tabular}

Fuente: Estadísticas Proyecto Creencias en Matemática.

Se puede observar que el 42\% (92 estudiantes) del total de la población está de "acuerdo" y "totalmente de acuerdo" en que el rendimiento académico en Matemática depende de la actitud del profesor hacia ellos y el 31\% (67 estudiantes) también del total de la población, está "totalmente en desacuerdo" y "en desacuerdo" con esta afirmación. Por otro lado, el $27 \%$ (6o estudiantes) está "ni de acuerdo ni en desacuerdo" con esta afirmación.
Si se analiza la opinión de los estudiantes, según la institución de procedencia, se observa que en el Liceo de Atenas el 32\% (21 estudiantes de la población de ese colegio) está de "acuerdo" y "totalmente de acuerdo" en que su rendimiento está influenciado por la actitud del profesor, mientras que en el Colegio Calasanz el porcentaje es del 48,5\% (34 estudiantes de la población de ese colegio) y en Liceo de San Isidro es de un 44\% (37 
estudiantes de la población de ese colegio).

En la tabla 6 se resumen las opiniones de los estudiantes con respecto a esta pregunta según sexo y nivel que cursan.

Tabla 6

Opinión de los estudiantes con respecto su rendimiento académico en relación con la actitud del profesor hacia ellos.

\begin{tabular}{|l|c|c|c|c|c|c|c|c|c|c|c|c|}
\hline Categorías & \multicolumn{4}{|c|}{ Octavo } & \multicolumn{4}{c|}{ Décimo } & \multicolumn{5}{c|}{ Total } \\
\hline & \multicolumn{3}{|c|}{ Masc. } & \multicolumn{1}{|c|}{ Feme. } & \multicolumn{2}{c|}{ Masc. } & \multicolumn{2}{c|}{ Feme. } & \multicolumn{2}{c|}{ Masc. } & \multicolumn{2}{c|}{ Feme. } \\
\cline { 2 - 17 } & $\mathrm{A}$ & $\%$ & $\mathrm{~A}$ & $\%$ & $\mathrm{~A}$ & $\%$ & $\mathrm{~A}$ & $\%$ & $\mathrm{~A}$ & $\%$ & $\mathrm{~A}$ & $\%$ \\
\hline $\begin{array}{l}\text { Totalmente de } \\
\text { acuerdo }\end{array}$ & 13 & 22 & 15 & 27 & 12 & 24 & 10 & 19 & 25 & 23 & 25 & 23 \\
\hline De acuerdo & 11 & 18 & 11 & 19 & 8 & 15 & 12 & 23 & 19 & 17 & 23 & 21 \\
\hline $\begin{array}{l}\text { Ni de acuerdo ni } \\
\text { en desacuerdo }\end{array}$ & 16 & 27 & 21 & 38 & 11 & 22 & 12 & 23 & 27 & 24 & 33 & 31 \\
\hline En desacuerdo & 8 & 13 & 4 & 7 & 14 & 27 & 12 & 23 & 22 & 20 & 16 & 15 \\
\hline $\begin{array}{l}\text { Totalmente en } \\
\text { desacuerdo }\end{array}$ & 12 & 20 & 5 & 9 & 6 & 12 & 6 & 12 & 18 & 16 & 11 & 10 \\
\hline Total & 60 & 100 & 56 & 100 & 51 & 100 & 52 & 100 & 111 & 100 & 108 & 100 \\
\hline
\end{tabular}

Fuente: Estadísticas Proyecto Creencias en Matemática.

Si se analiza la opinión de los estudiantes según el nivel matriculado, se tiene que el 40,7\% (42 estudiantes) de los alumnos de décimo año y el 43\% (50 estudiantes) de octavo, está de "acuerdo" y "totalmente de acuerdo" en que el rendimiento académico depende de la actitud del profesor.

Por otro lado, considerando la opinión de los estudiantes, según sexo, se puede notar que el 40\% (44 estudiantes) de los hombres y el $44 \%$ (48 estudiantes) de las mujeres está de "acuerdo" y "totalmente de acuerdo" en que la actitud del profesor sí influye en el rendimiento académico.

\section{Discusión y conclusiones}

En general, no hay una diferencia significativa entre las poblaciones de las tres instituciones según el sexo y nivel que cursan. El porcentaje de estudiantes según sexo es muy parecido en todas las instituciones, pues como se observa en la tabla uno, de los 219 estudiantes que participaron en la investigación, el 50,7\% (111 estudiantes) son hombres mientras que el 49,3\% (108 estudiantes) son mujeres, teniendo el Liceo de San Isidro la mayor matricula. De igual manera, la distribución según el nivel que cursan y tomando en cuenta la cantidad de estudiantes participantes (65 en el Liceo de Atenas, 70 en el Colegio 
Calasanz y 84 en el Liceo de San Isidro) se puede concluir que es muy parecida en los tres colegios, pues como se observa en la tabla uno en el Liceo de Atenas 32 estudiantes cursan octavo año mientras que 33 lo hacen en décimo, en el Colegio Calasanz 37 están en octavo y 33 en décimo y en el Liceo de San Isidro, 47 en octavo y 37 en décimo.

Se tendría que hacer otra investigación para determinar si existen otras diferencias entre estas tres instituciones, tomando en cuenta el estrato social del que provienen los estudiantes.

En lo que se refiere a la opinión que tienen los estudiantes de las clases de Matemáticas, llama la atención que un porcentaje bastante significativo está en "desacuerdo" y "totalmente en desacuerdo" en que estas lecciones son eternas y pesadas como se puede comprobar en la tabla dos. De igual manera, son los estudiantes varones (94 estudiantes del total de la población) los que más consideran que las lecciones de matemáticas no son eternas ni pesadas, en comparación con 68 estudiantes mujeres del total de la población que opinan lo mismo, caso contrario con los estudiantes de octavo año que no están ni de acuerdo ni en desacuerdo en que las clases de matemáticas son aburridas y pesadas como se muestra en la tabla tres.

La valoración que hacen principalmente los estudiantes varones de décimo año en relación a que las clases de matemáticas no son aburridas ni pesadas, tal vez se deba a que históricamente la sociedad le ha atribuido al hombre el desarrollo de la ciencia y la tecnología; de ahí que la elección de las carreras a seguir por los estudiantes del sexo masculino en secundaria son por lo general carreras que tienen mucha matemática, como: Ingenierías, Medicina, Física, Geología, Astronomía, entre otros, y a la mujer por el contrario, la sociedad le ha atribuido un conocimiento tácito que tiene que ver con el cuido de los hijos y la administración del hogar; así entonces, muchas mujeres se inclinan por carreras como: Educación Preescolar, Enfermerías, Educación Primaria, Psicología, por citar algunas, las cuales no tienen muchos cursos de matemáticas en su oferta académica.

También se puede concluir que muchas de las respuestas de los estudiantes se ubican en la categoría "ni de acuerdo ni en desacuerdo", esto se verifica con las opiniones que dieron ellos en relación con las clases de matemática; al respecto opinaron que es: aburrida, pesada, cansada, llena de trabajo, larga, repetitiva, tediosa, nunca se disfruta, muchos problemas, por citar algunas. Sin embargo para otros es: entretenida, mejor que las demás, divertida, retadora, buena y bonita.

Por otro lado, los estudiantes opinan que el profesor de matemáticas es aburrido, poco innovador, no es detallista, cansa escucharlo, explica muy rápido, no se le entiende, es injusto para evaluar, desinteresado por los alumnos y le brinda más atención a los que entienden. De igual manera, otros expresaron que es amable, bueno, paciente, amistoso y simpático. 
Es importante señalar que para los estudiantes que participaron de la investigación, su rendimiento académico sí depende de actitud que tenga el profesor hacia ellos, pues como se observa en la tabla cuatro un porcentaje muy significativo de los estudiantes está, "de acuerdo y totalmente de acuerdo" con esta afirmación. De los 65 estudiantes matriculados en el Liceo de Atenas, 21 (9 de octavo y 12 de décimo), en el Colegio Calasanz de los 70 estudiantes matriculados, 34 (20 de octavo y 14 de décimo) y en el Liceo de San Isidro de los 84 estudiantes matriculados, 37 (21 de octavo y 16 de décimo), así lo manifiestan.

De igual manera, tanto los hombres como las mujeres opinan que la actitud del profesor hacia ellos influye en su rendimiento académico, pues como se observa en la tabla cinco, el 42\% (92 estudiantes, 44 hombres y 48 mujeres) del total de la población está "totalmente de acuerdo y de acuerdo" con esta afirmación. Por otra parte, 50 de esos estudiantes cursan octavo año y 42 décimo.

Finalmente, de acuerdo con lo anterior, se puede concluir que el rendimiento académico en Matemática, sí depende de la actitud que tenga el profesor hacia ellos; no obstante es necesario investigar un poco más la manera cómo influye el docente en el rendimiento $y$ considerar otras variables de tipo económico, social y cultural, motivación de los estudiantes hacia la disciplina, estado de ánimo de los docentes, otras creencias hacia la disciplina, entre otras variables, que puedan influir en el trabajo escolar de los estudiantes.

\section{Referencias}

Barrantes, H. (2008). Creencias sobre las Matemáticas en Estudiantes de la Educación Secundaria Costarricense. Cuadernos de Investigación y Formación en Educación Matemática, 3 (4), 45-69

Barrantes, R. (2005). Investigación: Un camino al conocimiento. San José: UNED.

De Faria, E. (2007). Creencias y Matemáticas. San José Costa Rica: Centro de Investigaciones Matemáticas y Metamatemáticas. Recuperado el 23 de octubre de 2010, de: <http://cimm.ucr.ac.cr/cuadernos/cuaderno4/cuaderno4_c1.pdf>

Gómez, I. (2007). Sistema de Creencias en los alumnos de secundaria. Madrid, España: Universidad Complutense de Madrid.Recuperado el 23 octubre de 2010, de <http://www.ucm.es/BUCM/revistas/edu/11302496/articulos/RCEDo707220125A.PDF>

Gómez, I. (2000). Matemática Emocional: Los afectos en el aprendizaje matemático. Madrid, España: NARCEA, S.A.

González, C. (2010). Educar más allá de las creencias. Ponencia presentada en el seminario Pedagogía 3000. Barcelona, España.

Llinares, S. (1994). Conocimiento profesional del profesor de matemáticas: Conocimientos, creencias y contexto en relación a la noción de función. Departamento de Didáctica de las Matemáticas. Universidad de Sevilla: España.

Moreira, A. (2001). La clase de matemática centrada en los alumnos. Recuperado el 23 de octubre de 2010, de <http://personales.ya.com/casanchi/did/clasecentradao1.pdf> 
Olfos, R. (2001). Entendiendo la clase de matemática. Recuperado el 23 de octubre de 2010, de $<$ http://dialnet.unirioja.es/servlet/articulo?codigo=2147164>

Pólya, G. (1966).Matemáticas y razonamiento plausible. Madrid: Editorial Tecnos, S.A.

Red Armonía Mundial. Principios del Nuevo Paradigma. Recuperado el 23 de noviembre del 2010, de $<$ http://worldharmonynetwork.voila.net/Principios2_esp.html

\title{
Nota acerca del autor
}

\author{
MSc.Eugenio Rojas Mora
}

Escuela de Ciencias Exactas y Naturales de la UNED.

Programa de Enseñanza de la Matemática

Encargado de la cátedra de Cálculo

Correo electrónico: eurojas@uned.ac.cr

\author{
MSc.Ronald Sequeira Salazar \\ Escuela de Ciencias Exactas y Naturales de la UNED. \\ Programa de Enseñanza de la Matemática \\ Encargado de la cátedra de Geometría
}

Correo electrónico rsequeira@uned.ac.cr 